\title{
PARYLENE-STRENGTHENED THERMAL ISOLATION TECHNOLOGY FOR MICROFLUIDIC SYSTEM-ON-CHIP APPLICATIONS
}

\author{
Chi-Yuan Shih, Yang Chen and Yu-Chong Tai \\ Electrical Engineering Department, California Institute of Technology \\ MC 136-93, Pasadena, CA 91125 , USA, E-mail: cyshih@caltech.edu
}

\begin{abstract}
Here we reported a novel technology using parylene-cross-linking structure to achieve on-chip airgap thermal isolation for microfluidic system-on-chip (SOC) applications. Two applications based on this technology, on-chip continuous-flow polymerase chain reaction (PCR) and on-chip temperature gradient liquid chromatography (LC) were successfully demonstrated. Device thermal performance in each example was characterized. Results showed that our technology not only provides excellent on-chip thermal isolation but also its simplicity of integration with other on-chip components makes versatile microfluidic SOC applications feasible.
\end{abstract}

Keywords: Parylene, Thermal isolation, Microfluidic, On-chip, Chromatography

\section{INTRODUCTION}

Silicon is a fundamental structure material in MEMS with a very high thermal conductivity of 148 $\mathrm{W} / \mathrm{m}-\mathrm{K}$ (compared with $73 \mathrm{~W} / \mathrm{m}-\mathrm{K}$ of iron). This specific thermal property guarantees great on-chip temperature uniformity, however it also generates design complexity for applications where multiple temperature zones are required on a single silicon chip. Some representative $\mathrm{SOC}$ applications that require multiple on-chip temperature zones are polymerase chain reaction [1] and temperature gradient liquid chromatography [2]. Ideally, for best temperature uniformity on each temperature zone, no silicon connection should remain between temperature zones and therefore a wafer-thickness-deep, temperaturezone-surrounding themal isolation structure needs to be used on the chip. While several thermal isolation technologies have been developed, the aerogel or porous-silicon structure in general provides only vertical direction thermal isolation [3]; floating membrane structure which uses thin dielectric layer to hold freestanding temperature zone is mechanically fragile and cannot stand much stress and strain encountered during device fabrication or testing procedures [4]; oxide-filled trench structure has only limited thermal isolation efficiency due to the still high thermal conductivity of oxide $(1.4 \mathrm{~W} / \mathrm{m}-\mathrm{K}$ for silicon dioxide) and the limited oxide thickness [5]. To attack these shortcomings, we propose here to use a novel parylene-cross-linking thermal isolation technology.

In the past decade, parylene was used extensively as a MEMS structure material to build microfluidic system components including sensors and actuators [69]. Because parylene is a flexible polymer material (an elongation break of $200 \%$ ) and has a very low thermal conductivity of $0.08 \mathrm{~W} / \mathrm{m}-\mathrm{K}$ (compared with 0.03 $\mathrm{W} / \mathrm{m}-\mathrm{K}$ of static air), using parylene to build thermal isolation structure can provide crucial benefits such as robust mechanical support to the temperature zones and high thermal isolation efficiency, which in general cannot both be provided at the same time by other thermal isolation technologies. As will be shown below, we have successfully developed the parylenestrengthened thermal isolation technology and demonstrated its applications, in the field of microfuidic system-on-chip.

\section{DESIGN AND FABRICATION}

Fig. 1 shows the process flow of the proposed parylene-strengthened thermal isolation technology.

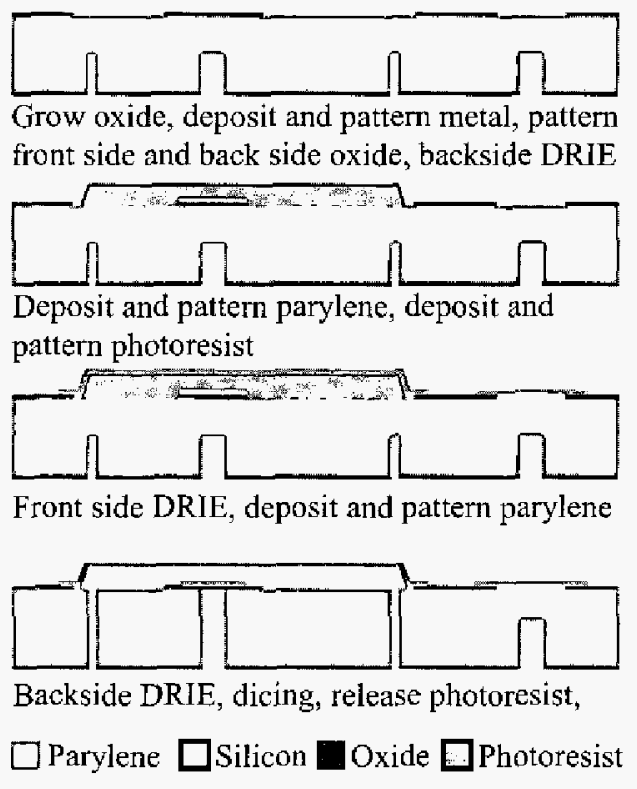

Fig. 1: Process flow of parylene-strengthened thermal isolation technology. 
This technology not only provides lateral on-chip thermal isolation but also it allows microfluidic channel and electrical path connection between temperature zones. Process starts with growing $1 \mu \mathrm{m}$ $\mathrm{SiO}_{2}$ layer on both sides of 4 -inch silicon wafers (thickness: $525+/-25 \mu \mathrm{m}$ ) by thermal oxidation. Metal is then deposited and pattemed on the wafer front side. Front side oxide is patterned with buffer HF (Transene, Danvers, MA). Backside oxide is then patterned to define the $100 \mu \mathrm{m}$-diameter liquid access holes and $150 \mu \mathrm{m}$-wide air gap for thermal isolation. Without removing photoresist on the backside oxide, DRIE (deep reactive ion etching) is used to etch backside silicon for $450 \mu \mathrm{m}$. 4um-thick parylene-C (Specialty Coating Systems, Indianapolis, IN) is then deposited and pattemed with oxygen plasma on wafer front side. $25 \mu \mathrm{m}$ photoresist AZ4620 (Clariant, Somerville, NJ) is deposited and patterned on wafer front side to form microfluidic channel sacrificial layer. A $100^{\circ} \mathrm{C}-3 \mathrm{hr}$ baking is carried out to avoid photoresist bubbling from latter thermal treatment. Front side oxide and photoresist are then used together as DRIE mask to etch down silicon for a $30 \mu \mathrm{m}$-deep, $15 \mu \mathrm{m}$ wide trenches with a mushroom profile in the trench bottom (by a modified DRIE Bosch process [10]). The trench-mushroom structure here is used as the anchoring space for parylene thin film deposition. 10 $\mu \mathrm{m}$ parylene is then conformally deposited on wafer front side, filling up the trenches and covering photoresist structures. Parylene is then patterned with oxygen plasma. DRIE is carried out on wafer backside again to etch through the silicon wafer for the liquid access holes and themal-isolation air gaps. Finally, the wafer is diced into $1.1 \mathrm{~cm} \times 1.1 \mathrm{~cm}$ chips and photoresist inside the column is dissolved by $80^{\circ} \mathrm{C}$ $24 \mathrm{hr}$ acetone bath through backside liquid access holes.

Fig. 2a shows the schematic plot of parylene-crosslinking structure. Parylene layer is deposited into silicon trenches created by DRIE to provide robust parylene anchoring. Since parylene has a high elongation break and a very low thermal conductivity, parylene-cross-linking structure provides a durable
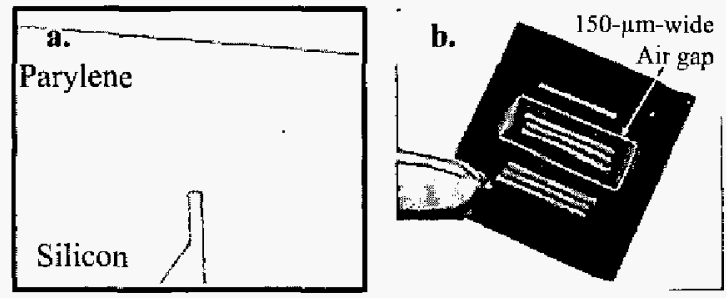

Fig. 2: a. Illustration of parylene-cross-linking structure, b. a photograph showing 150um-wide through-wafer air gap and the silicon temperature zone (center piece).

mechanical support to temperature zones while not much heat will be conducted through the structure itself. Fig. $2 b$ shows a silicon temperature zone defined by $150 \mu \mathrm{m}$-wide air gap. Fig. 3a shows the cross-sectional view of the anchored parylene column which is $100 \mu \mathrm{m}$-wide and $25 \mu \mathrm{m}$-high. Fig. $3 \mathrm{~b}$ shows the fabricated microfluidic channel on top of a parylene membrane nunning across the thermal isolation air gap from one temperature zone to the other.
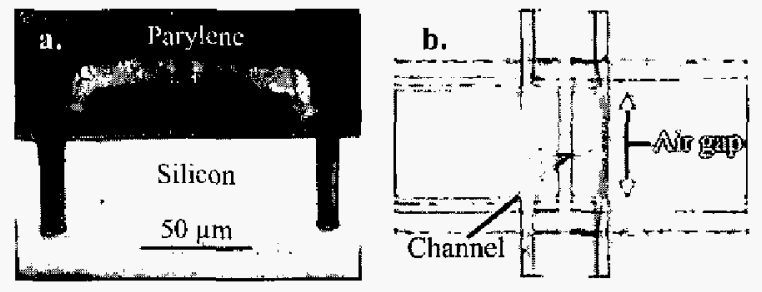

Fig. 3: a. Cross-sectional view of the anchored parylene column, b. fabricated microfluidic channel running across thermal isolation air gap.

\section{APPLICATION AND DISCUSSION}

Two examples of microfluidic SOC application using proposed parylene-strengthened thermal isolation technology were successfully carried out as follows.

\section{On-chip continuous-flow PCR}

Polymerase chain reaction requires the DNA sample solution to periodically pass through three temperature zones including the high temperature zone $\left(94^{\circ} \mathrm{C}\right)$ for double-strand DNA (dsDNA) melting, the low temperature zone $\left(60^{\circ} \mathrm{C}\right)$ for primer annealing and the medium temperature zone $\left(72^{\circ} \mathrm{C}\right.$ ) for primer extension [1]. With the developed thermal isolation technology, three temperature zones defined by air gaps were created on the silicon substrate. Each temperature zone had its individual resistive heater for precise temperature control. As shown in Fig. 4a, $10 \mathrm{PCR}$ cycles were prepared on a $0.8 \mathrm{~cm} \times 0.8 \mathrm{~cm}$ chip area. $\mathrm{PCR}$ time ratio among the three temperature zones was defined by the fluidic channel length in each temperature zone. Fig. $4 b$ then shows the thermal image of the powered up PCR temperature zones taken by InfraScope ${ }^{\mathrm{TM}}$ system.

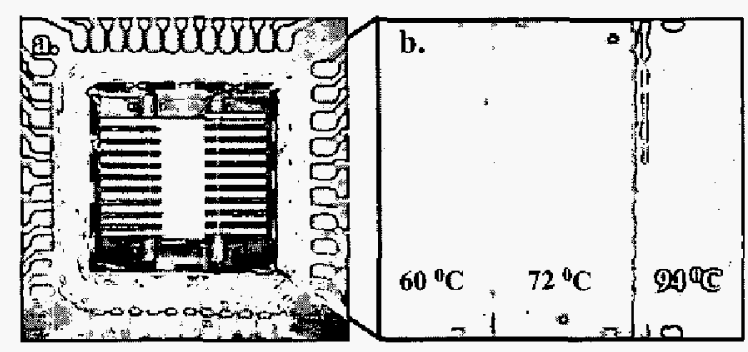

Fig. 4: a. Continuous-flow $P C R$ device with $10 P C R$ cycles, b. thermal image of a powered up PCR device., temperature was read out from heater resistance via $T C R$ (temperature coefficient of resistance) data. 
On-chip temperature gradient liquid chromatography MEMS technology is a fundamental tool to carry out the miniaturization goal of modern HPLC system [11].
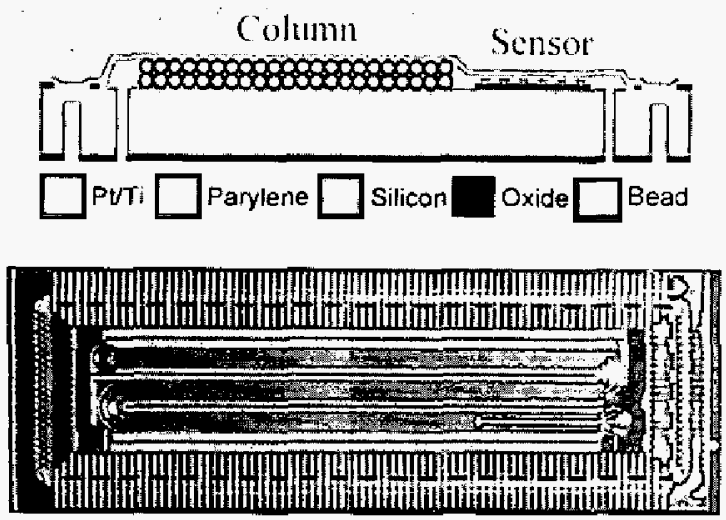

Fig. 5: On-chip temperature gradient liquid chromatography device. Dashed rectangle defines the separation column zone surrounded by $150 \mu \mathrm{m}$-wide air gap; thin white lines are parylene cross-linking structure; running between the separation column is the resistive heater.

While most researchers have pursued solventcontrolled MEMS LC [12], temperature gradient LC (i.e., changing the LC column's temperature as a function of time to achieve analytes elution) has never been demonstrated in MEMS before [13]. In fact, we believe MEMS temperature gradient LC is extremely attractive because of the ease of achieving on-chip temperature control by MEMS [14]. Based on this motivation, we used the proposed process flow to fabricate the first chip-based temperature gradient LC system which integrated LC column, electrochemical

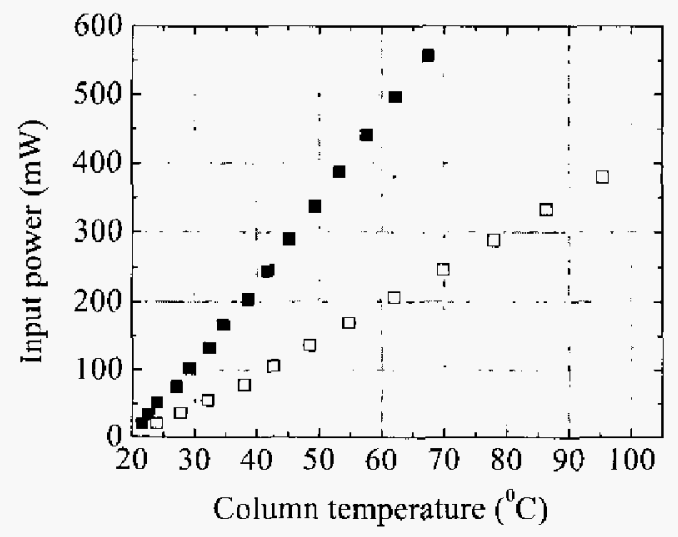

Fig. 6: Thermat isolation efficiency: (a) column without thermal isolation structure, () column surrounded by parylene-strengthened air-gap thermal isolation structure. Thermal isolation structure effectively reduced column-zone heating power consumption by $58 \%$. sensor, resistive heater and parylene-strengthened thermal isolation structure on a single silicon chip as shown in Fig. 5.

Thermal isolation efficiency of the parylenestrengthened air-gap structure was then studied. Results showed that the thermal isolation structure effectively reduced column-zone heating power consumption by $58 \%$ (Fig. 6) and the off-column temperature rise (compared with on-column temperature rise) by $67 \%$ (Fig. 7). With the offcolumn cooling function available, it is feasible to layout the electrochemical sensor in the cooling area when analyte sensing needs to be done at low background temperature. Chip thermal performance can be further improved by reducing the temperaturezone area or increasing the air-gap width. Fig. 8 then shows the successful separation and detection of derivatized amino acids using our chip-based temperature gradient LC system [15].

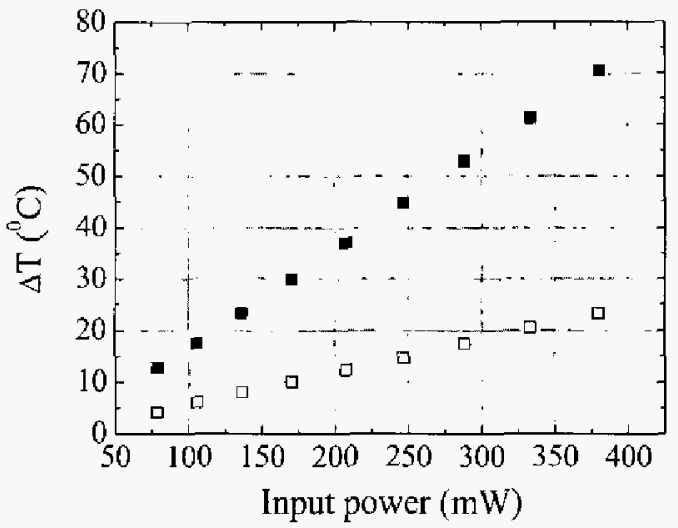

Fig. 7: On-chip temperature rise ( $\Delta T)$ spatial distribution during temperature gradient operation: (i) air-gap-surrounded column area, () off-column area. Compared with on-coltumn area, temperature rise in the off-column area was reduced by $67 \%$

\section{CONCLUSION}

A novel thermal isolation technology using parylene-cross-linking structure to achieve on-chip air gap thermal isolation was demonstrated. Two applications based on this technology, on-chip continuous-flow polymerase chain reaction and onchip temperature gradient liquid chromatography were successfully carried out. Devices thermal performance was characterized. Results showed that our technology not only provides excellent on-chip thermal isolation efficiency but also its simplicity of integration with other on-chip components makes versatile microfluidic SOC applications feasible. Future work is focused on improving fluidic column structure robustness especially around the air gap region so to make more high pressure applications feasible. 


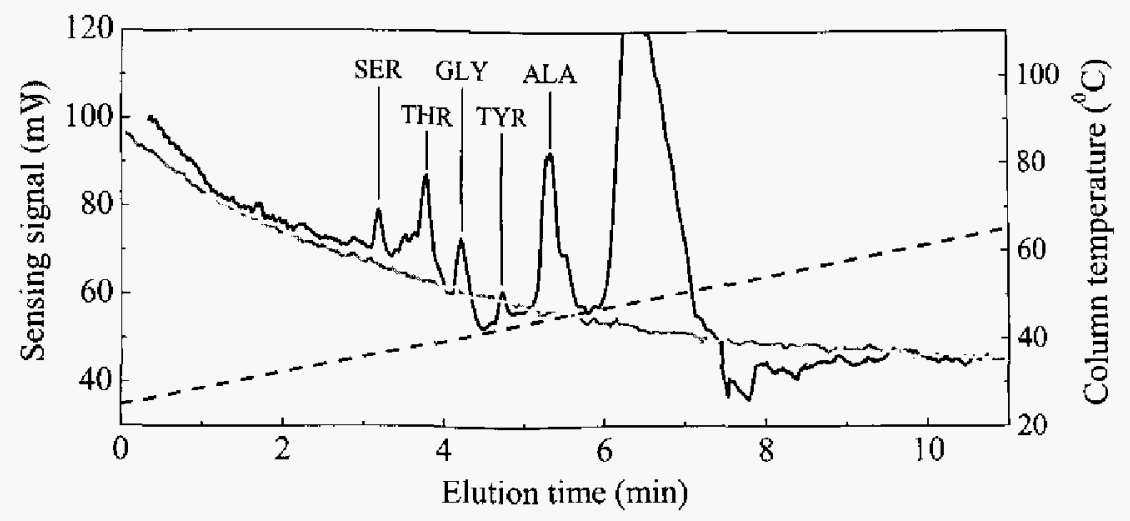

Fig. 8: Chromatogram showing derivatized amino acids separated using temperature gradient elution: (black line) temperature gradient elution with column temperature scanning from $25^{\circ} \mathrm{C}$ at 0 min to $65^{\circ} \mathrm{C}$ at 11 min with a slope of $3.6^{\circ} \mathrm{C}$ min, (gray line) isothermal elution with column temperature fixed at $25^{\circ} \mathrm{C}$ throughout the separation, (dash line) temporal temperature gradient which was applied to the column during separation.

\section{ACKNOWLEDGEMENTS}

This work was supported by the NSF Center for Neuromorphic Systems Engineering (CNSE) at Caltech. The authors would also like to thank Mr. Trevor Roper for his assistance in processing.

\section{REFERENCES}

[1] M.U. Kopp, A.J. Mello and A. Manz, "Chemical amplification: continuous-flow PCR on a chip," Science, 280, 1046-1048 (1998).

[2] T. Greibrokk, "Heating or cooling your column in liquid chromatography," Anal. Chem., 74, 374A378A (2002).

[3] J.A. Ruffner, P.G. Clem, B.A. Tuttle, C.J. Brinker, C.S. Sriram and J.A. Billington, "Uncooled thin film infrared imaging device with aerogel thermal isolation deposition and planarization techniques," Thin Solid Films, 332, 356-361 (1998).

[4] B.W. Oudheusden and A.W. Herwaarden, "Highsensitivity 2-D flow sensor with an etched thermal isolation structure," Sensors and Actuators, A21-23, 425-430 (1990).

[5] R. Kersjes and W. Mokwa, "A fast liquid flow sensor with thermal isolation by oxide-filled trenches," Sensors and Actuators, A46-47, 373-379 (1995).

[6] X.Q. Wang, Q. Lin and Y.C. Tai, "A parylene micro check valve," the $12^{\text {th }}$ IEEE International Conference on Micro Electro Mechanical Systems, (MEMS '99), Florida, USA, 177-182, Jan. 1999.

[7] L. Licklider, X.Q. Wang, A. Desai, Y.C. Tai and T.D. Lee, "A micromachined chip-based electrospray source for mass spectrometry," Anal. Chem., 72, 367-375 (2000).

[8] J.R. Webster, M.A. Burns, D.T. Burke and C.H.
Mastrangelo, "An inexpensive plastic technology for microfabricated capillary electrophoresis chips," Micro Total Analysis Systems '98, Banff, Canada, 249-252, Oct. 1998.

[9] C. Grojean, X. Yang and Y.C. Tai, "A thermopneumatic peristaltic micropump," 1999 International Conference on Solid-State Sensors and Actuators (Transducers '99), Sendai, Japan, 1776-1779, Jun. 1999.

[10] M. Liger, D.C. Rodger and Y.C. Tai, "Robust parylene-to-silicon mechanical anchoring," the 16th IEEE International Conference on MicroElectroMechanical Systems (MEMS2003), Kyoto, Japan, 602-605, Jan. 2003.

[11] V.R. Meyer, "Practical High-Performance Liquid Chromatography", John Wiley \& Sons, 1999, 310311 .

[12] J. Xie, J. Shih, Q. He, C. Pang, Y.C. Tai, Y.N. Miao and T.D. Lee, "An integrated LC-ESI chip with electrochemical-based gradient generation," the 17th IEEE International Conference on MicroElectroMechanical Systems (MEMS 2004), Maastricht, The Netherlands, 334-337, Jan. 2004.

[13] C.Y. Shih, Y. Chen, J. Xie, Q. He and Y.C. Tai, "On-chip temperature gradient liquid chromatography," the 18th IEEE International Conference on MicroElectroMechanical Systems (MEMS 2005), Miami, USA, 782-785, Jan. 2005.

[14] C.N. Liu, N.M. Toriello, R. Maboudian and R.A. Mathies, "High throughput polymerase chain reaction-capillary array electrrophoresis (PCRCAE) microchip," Micro Total Analysis Systems 2004, Malmo, Sweden, 297-299, Sept. 2004.

[15] B.W. Boyd, S.R. Witowski and R.T. Kennedy, "Trace-level amino acid analysis by capillary liquid chromatography and application to in vivo microdialysis sampling with 10 -s temporal resolution," Anal. Chem., 72, 865-871 (2000). 